\title{
Recurrent Disturbance of Consciousness in a Peritoneal Dialysis Patient with Severe Hypercalcemia Caused by Daily Low-Dose Keto Acids Supplementation
}

\author{
Peng Li, Xiaojing Zhong and Jianying Niu*
}

Renal Division, Shanghai Fifth People's Hospital, Fudan University, Shanghai, People's Republic of China

"Corresponding author: Jianying Niu, Renal Division, Shanghai Fifth People's Hospital, Fudan University, 801 Heqing Road, Minhang District, Shanghai, People's Republic of China, Tel: 021-24289516; Fax: 021-24289902; E-mail: njyphd2008@163.com

Received date: December 13, 2018; Accepted date: December 21, 2018; Published date: December 28, 2018

Copyright: (c) $2018 \mathrm{Niu} \mathrm{J}$ et al. This is an open-access article distributed under the terms of the Creative Commons Attribution License, which permits unrestricted use, distribution, and reproduction in any medium, provided the original author and source are credited.

\begin{abstract}
Keto acids is widely used in dialysis patients with end-stage renal disease, which can effectively correct hypocalcemia, reduce blood PTH as well as blood phosphorus, and elevate blood albumin. Keto acids have a very low incidence of side effects. So far, no cases of Keto acids-induced severe hypercalcemia had been reported. The authors here present a case of continuous ambulatory peritoneal dialysis (CAPD) patient who repeatedly developed severe hypercalcemia accompanied by consciousness disturbance due to daily low-dose keto acids administration. In this case, common causes of hypercalcemia were excluded by thorough physical examination and investigations. The blood calcium concentration of this patient had reached $3.83 \mathrm{mmol} / \mathrm{L}$, belonging to severe hypercalcemia, and the clinical manifestations of cognitive disorder required urgent treatment. After the suspension of keto acids, the serum calcium concentration returned to normal, and the peritoneal dialysis scheme resumed as normal after discharge, which could still maintain the normal calcium concentration. When the patient took the medicine again, severe hypercalcemia occurred as expected. After drug withdrawal, blood calcium backed to normal level, and blood phosphorus increased, which further confirmed that calcium and phosphorus metabolism disorder was closely related to the use of keto acids. This case here indicate that close monitoring of blood calcium, phosphorus and other indicators are needed, in order to prevent potential risk of hypercalcemia which would endanger the safety of patient's life.
\end{abstract}

Keywords: Disturbance of consciousness; Peritoneal dialysis; Hypercalcemia; Keto acids

\section{Introduction}

Peritoneal dialysis patients who prescribed a low-protein diet supplemented with keto acids had showed a better preservation of residual renal function (RRF), lower serum phosphorus level, as well as lower intact parathyroid hormone (iPTH) level [1].Moreover, improvements in hs-CRP and the leptin/adiponectin ratio (LAR) in continuous ambulatory peritoneal dialysis (CAPD) patients with Keto acids administration, suggested that metabolic benefit would also achieved by the therapy [2]. However, higher serum calcium levels would be observed during follow-up, as the keto acid tablets contain 50 $\mathrm{mg}$ of calcium each. So caution on the possible risk for severe hypercalcemia in CAPD patients treated with keto acids is definitely needed. Considering researches focusing this are limited, in this paper, the author shared a case of CAPD patient who repeatedly developed severe hypercalcemia accompanied by consciousness disturbance due to taking daily low-dose keto acids.

\section{Case Report}

A 73-year-old Chinese non-diabetic female patient $(74 \mathrm{~kg}$ ) was admitted in July 2017 because of an altered level of consciousness for one week. The patient was a known case of chronic kidney disease 5 years ago and treated with CAPD (Tenckhoff double-cuff catheter) for 3 years. She also had histories of hypertension, and a transient ischemic attack in 2014. There is no prior history of catheter infection,
CAPD peritonitis. Her PD regimen was: $1.5 \%$ Low calcium $(1.25$ $\mathrm{mmol} / \mathrm{L}$ ) dextrose dialysate (Dianeal'; BaxterChina Ltd, Guangzhou, China) $2 \mathrm{~L}\left(4 \mathrm{~h}^{\star} 1\right.$ time $)+2.5 \%$ Low calcium dextrose dialysate $2 \mathrm{~L}\left(4 \mathrm{~h}^{\star} 2\right.$ times) with Twin Bag system (UltraBag; Baxter China Ltd). Her existing list of regular oral medications included amlodipine $5 \mathrm{mg}$ daily, atorvastatin $20 \mathrm{mg}$ daily, keto acids (Ketosteril'; Fresenius-Kabi Co., Ltd, Beijing, China) $2,520 \mathrm{mg}$ with three meals, without taking active vitamin $\mathrm{D}$ and calcium-containing phosphorus binder. On presentation, her blood pressure was $134 / 75 \mathrm{~mm}$ of $\mathrm{Hg}$, pulse rate 84 per minute, and core temperature 97.7degrees Fahrenheit. Pupils were 4 millimeters in size, equal, with no scleral icterus or conjunctivitis. Cardiac exam revealed no jugular venous distention, tachycardia or murmur. Lungs were clear to auscultation bilaterally. Abdomen was soft with normal bowel sounds and no hepatosplenomegaly. Extremities were without peripheral edema. There were no definite focal neurological signs in the patient and the Glasgow Coma Score was 9 . The preliminary laboratory findings were: serum creatinine 8.33 $\mathrm{mg} / \mathrm{dL}$, serum urea nitrogen $55.57 \mathrm{mg} / \mathrm{dL}$, serum adjusted calcium 3.30 $\mathrm{mmol} / \mathrm{L}$, serum phosphorus $1.05 \mathrm{mmol} / \mathrm{L}$, serum albumin $44.8 \mathrm{~g} / \mathrm{L}$, serum potassium $3.4 \mathrm{mmol} / \mathrm{L}$, serum magnesium $1.46 \mathrm{mmol} / \mathrm{L}$, serum iPTH $33.9 \mathrm{pmol} / \mathrm{L}$, blood hemoglobin $142 \mathrm{~g} / \mathrm{L}$, blood platelet count $145,000 / \mathrm{uL}$. The Kt/Vtotal was $1.63 /$ week. There was no abnormality detected in the computed tomography (CT) of the brain.

Treatment to improve the adequacy of peritoneal dialysis, and increase calcium efflux from PD fluid, her PD regimen was adjusted to: $1.5 \%$ Low calcium $(1.25 \mathrm{mmol} / \mathrm{L})$ dextrose dialysate $2 \mathrm{~L}\left(4 \mathrm{~h}^{\star} 2\right.$ times) $+2.5 \%$ Low calcium dextrose dialysate $2 \mathrm{~L}\left(4 \mathrm{~h}^{\star} 2\right.$ times $)+1.5 \%$ low calcium dextrose dialysate $2 \mathrm{~L}$ overnight. Keto acids administration was 
Page 2 of 3

stopped and $500 \mathrm{~mL}$ of normal saline was dropped daily. After treatment, the patient showed improvement in reaction retardation, fatigue and mental malaise. After 4 days of hospitalization, test results were as follows: serum adjusted calcium $2.91 \mathrm{mmol} / \mathrm{L}$, serum phosphorus $1.32 \mathrm{mmol} / \mathrm{L}$, serum magnesium $1.45 \mathrm{mmol} / \mathrm{L}$. The patient and her family refused further test to determine the cause of hypercalcemia. Therefore, the patient was discharged from the hospital. Two weeks after discharge, the test results: blood calcium 2.47 $\mathrm{mmol} / \mathrm{L}$, blood phosphorus $2.19 \mathrm{mmol} / \mathrm{L}$. But one month after discharge, the patient was admitted again for fatigue and slow reaction for 1 day. The patient's family member had given her keto acids 2,520 $\mathrm{mg}$ with three meals again. The serum calcium was over $3.49 \mathrm{mmol} / \mathrm{L}$ in the emergency room. Her PD regimen was immediately adjusted to: $1.5 \%$ Low calcium dextrose dialysate $2 \mathrm{~L}$ ( $3 \mathrm{~h}^{\star 5}$ times). A single 50IU dose of salmon calcitonin intramuscular injection daily and $500 \mathrm{ml}$ of normal saline daily were given to treat hypercalcemia. The next day, the serum adjusted calcium was $3.83 \mathrm{mmol} / \mathrm{L}$, and serum phosphorus was $0.43 \mathrm{mmol} / \mathrm{L}$. Other detailed examination results were: iPTH 43.4 $\mathrm{pmol} / \mathrm{L}, 25$ hydroxy vitamin D $11.02 \mathrm{nmol} / \mathrm{L}$, calcitonin $7.4 \mathrm{pg} / \mathrm{mL}$. Tumor markers (AFP, CEA, CA125, CA153, NSE, CA199, CA724, CA50, CA211, CA242) were normal. No obvious abnormalities were observed in $99 \mathrm{mTc}$-MIBI static imaging of parathyroid gland. Reexamination results on the fourth day after admission were: serum calcium $2.35 \mathrm{mmol} / \mathrm{L}$. The patient returned to normal without symptoms of fatigue and slow reaction and was discharged from hospital.

Follow-up after discharged from hospital, the patient's serum calcium was maintained at $2.48 \mathrm{mmol} / \mathrm{L} \sim 2.6 \mathrm{mmol} / \mathrm{L}$ during longterm follow-up (Figure 1 for the changes of serum calcium and phosphorus value).

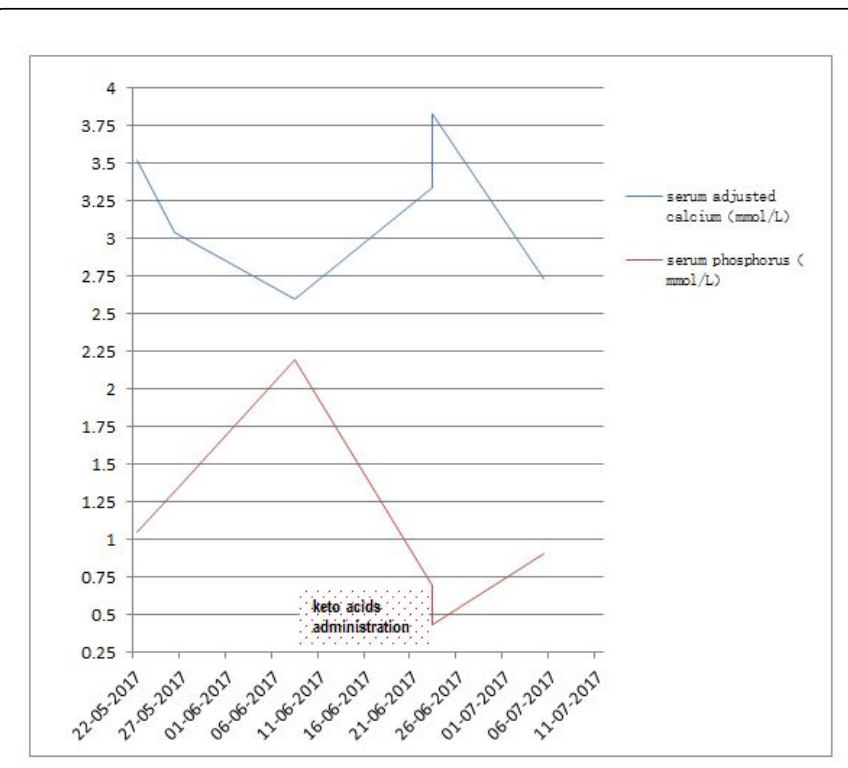

Figure 1: Changes of serum calcium and phosphorus value.

\section{Discussion}

Keto acids have a very low incidence of side effects and no cases of Keto acids-induced severe hypercalcemia had been reported in literature before. If hypercalcemia occurs, it is recommended to reduce vitamin D intake. When hypercalcemia persists, reduce the dosage of this product and avoid intake of other calcium-containing substances.

However, the assessment of calcium balance in dialysis patients (including PD patients) becomes extremely complex. It has to take into account not only dietary calcium intake, calcium supplement dose, intake of vitamin D analogs, stool calcium output, calcium uptake by soft tissue, urinary calcium excretion, continuous calcium flux from the exchangeable calcium bone pool, and net bone remodeling or turnover, but also calcium influx and efflux from PD fluid, depending on the dialysate calcium concentration and the amount of calcium removed with dialysis ultrafiltration [3-6].

Hypercalcemia can be diagnosed when blood calcium value is more than $2.6 \mathrm{millimol} / \mathrm{l}$ with serum protein concentration in the normal range. If accompanied by hyperalbuminemia, hypoalbuminemia or significantly increased platelet count, ionic calcium should be determined for definite diagnosis and combined with clinical manifestations of hypercalcemia. In this case, the serum albumin and blood platelet count were in the normal range, and pseudohypercalcemia could be excluded. The clinical score of hypercalcemia is as follows: mild $(<3.0 \mathrm{mmol} / \mathrm{L})$, often asymptomatic, generally without urgent treatment. Moderate (3.0-3.5 mmol/L), if the increase rate of blood calcium is slow, the patient can often tolerate it, but need prompt treatment. Severe $(>3.5 \mathrm{mmol} / \mathrm{L})$, prone to arrhythmia or coma requiring urgent treatment [7]. The blood calcium concentration of this patient had reached $3.83 \mathrm{mmol} / \mathrm{L}$, belonging to severe hypercalcemia, and the clinical manifestations of cognitive disorder required urgent treatment. Of course, clinical manifestations depend not only on calcium concentration, but also on the rate of calcium increase.

After a definite diagnosis, the cause of the disease should be actively sought. Human calcium balance regulation mainly depends on the action of parathyroid hormone, vitamin $\mathrm{D}$ and calcitonin on bone, kidney and digestive tract. According to statistics, $90 \%$ of hypercalcemia is caused by primary hyperparathyroidism or malignant tumors. Relatively rare causes include: thiazide diuretics, familial low urinary calcium hypercalcemia, non-malignant granulomatous disease, thyrotoxicosis, too much vitamin D, rhabdomyolysis, adrenocortical function decline, hypervitaminosis A, theophylline intoxication, pheochromocytoma, etc. [7].

In our case, common causes of hypercalcemia were excluded by thorough physical examination and investigations. After the elimination of other causes, it was speculated that hypercalcemia was related to the use of keto acids tablets. This medicine contains four kinds of calcium ketone ammonia and one kind of calcium hydroxy ammonia. Each tablet contains $50 \mathrm{mg}$ of calcium which means taking 12 tablets daily would increases the intake of calcium by $600 \mathrm{mg}$. This is further supported by the fact that no further episodes of consciousness disturbance occurred following keto acids discontinuation. Importantly, no adjustments were made to her other regular oral medications. After the suspension of the drug, the serum calcium concentration returned to normal, and the peritoneal dialysis scheme resumed as normal after discharge, which could still maintain the normal calcium concentration. When the patient took the medicine again, severe hypercalcemia occurred as expected. After keto acids discontinuation, hypercalcemia can be recovered, which also proves that hypercalcemia is related to the medicine. At the same time, when the serum calcium increased significantly after taking this medicine, the serum phosphorus decreased significantly, which is related to the elimination of phosphorus by the drug in the intestinal 
Citation: Li P, Xiaojing Z, Niu J (2018) Recurrent Disturbance of Consciousness in a Peritoneal Dialysis Patient with Severe Hypercalcemia Caused by Daily Low-Dose Keto Acids Supplementation. J Nephrol Ther 8: 322. doi:10.4172/2161-0959.1000322

Page 3 of 3

tract. After drug withdrawal, blood calcium returned to normal, and blood phosphorus increased, which further confirmed that calcium and phosphorus metabolism disorder was closely related to the use of the drug. These aforementioned factors cumulate in a 'probable' rated adverse drug reaction (ADR), assessed objectively by the Naranjo probability algorithm for ADR [8].

\section{Conclusion}

Keto acids are widely used in dialysis patients with end-stage renal disease $[9,10]$. The drug can effectively correct hypocalcemia, reduce blood PTH, elevate blood albumin and reduce blood phosphorus, so as to protect the patient's residual renal function. However the case here indicate that close monitoring of blood calcium, phosphorus and other indicators are needed, in order to prevent hypercalcemia which would endanger the safety of patient's life.

\section{Acknowledgement}

The authors thank all PD nurses, technicians, and staff in the Department of Renal Division, Shanghai Fifth People's Hospital, Fudan University, for their contributions.

\section{Author Contributions}

Peng Li, Xiaojing Zhong and Jianying Niu were physicians for the patient, and Peng Li wrote the manuscript and carried out final preparation of the manuscript. All authors carried out the literature review and drafted the manuscript. All authors read and approved the final manuscript.

\section{References}

1. Jiang N, Qian J, Sun W, Lin A, Cao L, et al. (2009) Better preservation of residual renal function in peritoneal dialysis patients treated with a lowprotein diet supplemented with keto acids: a prospective, randomized trial. Nephrol Dial Transplant 24: 2551-2558.

2. Dong J, Li YJ, Xu R, Ikizler TA, Wang HY (2015) Ketoacid Supplementation Partially Improves Metabolic Parameters in Patients on Peritoneal Dialysis. Perit Dial Int 35: 736-742.

3. Bushinsky DA (2010) Contribution of intestine, bone, kidney, and dialysis to extracellular fluid calcium content. Clin j Am Soc Nephrol 5 Suppl 1: S12-22.

4. Moe SM (2010) Confusion on the complexity of calcium balance. Semin Dial 23:492-497.

5. Peacock M (2010) Calcium metabolism in health and disease. Clin j Am Soc nephrol 5(Suppl 1): S23-30.

6. Wang AY (2014) Calcium balance and negative impact of calcium load in peritoneal dialysis patients. Perit Dial Int 34: 345-352.

7. Walsh J, Gittoes N, Selby P (2016) Society For Endocrinology Endocrine Emergency Guidance: Emergency management of acute hypercalcaemia in adult patients. Endocr Connect 5: 9-11.

8. Naranjo CA, Busto U, Sellers EM, Sandor P, Ruiz I, et al. (1981) A method for estimating the probability of adverse drug reactions. Clin Pharmacol Ther 30: 239-245.

9. Jiang N, Qian J, Lin A, Fang W, Cao L, et al. (2010) Improved plasma amino acids pattern following 12 months of supplemented low-protein diet in peritoneal dialysis patients. Ren Fail 32: 709-715.

10. Li H, Long Q, Shao C, Fan H, Yuan L, et al. (2011) Effect of Short-Term Low-Protein Diet Supplemented with Keto Acids on Hyperphosphatemia in Maintenance Hemodialysis Patients. Blood Purif 31: 33-40. 